\title{
Elevated L-threonine is a biomarker for Lassa fever and Ebola
}

\author{
Trevor V. Gale ${ }^{1,6}$, John S. Schieffelin², Luis M. Branco ${ }^{3}$, Robert F. Garry ${ }^{1,3^{*}}$ (I) and Donald S. Grant ${ }^{4,5^{*}}$
}

\begin{abstract}
Background: Lassa fever and Ebola are characterized by non-specific initial presentations that can progress to severe multisystem illnesses with high fatality rates. Samples from additional subjects are examined to extend and corroborate biomarkers with prognostic value for these diseases.

Methods: Liquid Chromatography Mass Spectrometry metabolomics was used to identify and confirm metabolites disrupted in the blood of Lassa fever and Ebola patients. Authenticated standards are used to confirm the identify of key metabolites.
\end{abstract}

Results: We confirm prior results by other investigators that the amino acid L-threonine is elevated during Ebola virus infection. L-Threonine is also elevated during Lassa virus infection. We also confirmed that platelet-activating factor (PAF) and molecules with PAF moiety are reduced in the blood of patients with fatal Lassa fever. Similar changes in PAF and PAF-like molecules were not observed in the blood of Ebola patients.

Conclusions: Metabolomics may provide tools to identify pathways that are differentially affected during viral hemorrhagic fevers and guide development of diagnostics to monitor and predict outcome.

Keywords: Lassa fever, Ebola, Viral hemorrhagic fevers, Liquid Chromatography Mass Spectrometry, L-Threonine, Metabolomics

\section{Background}

Select features within the metabolome may serve as biomarkers for disease severity/progression/outcome and lend themselves to the design of prognostic methods for viral diseases, such as Lassa fever and Ebola. We have previously characterized endogenous small molecules with prognostic value originating from the blood of febrile patients triaged to the Lassa fever ward in Kenema, Sierra Leone [1]. In an extraordinary multi-omics study Eisfeld and coworkers [2] demonstrated that levels of serum L-threonine were elevated in the blood of patients with Ebola, another severe viral hemorrhagic

*Correspondence: rfgarry@tulane.edu; donkumfel@yahoo.co.uk

${ }^{1}$ Department of Microbiology and Immunology, Tulane University, 1430

Tulane Avenue, JBJ568, New Orleans, LA 70112, USA

${ }^{4}$ Viral Hemorrhagic Fever Program, Kenema Government Hospital, Kenema, Sierra Leone

Full list of author information is available at the end of the article fever (VHF). Prior research has shown that L-threonine has significance as a biomarker in infectious disease and inflammation. Previously, L-threonine has been reported as a biomarker for both tuberculosis [3] and malaria [4]. Our prior study identified multiple unknown metabolites as potential biomarkers of acute Lassa fever [1]. Inspired by the results of Eisfeld and coworkers [2] we corroborate $\mathrm{L}$-threonine as a biomarker of acute Lassa fever. We also contrast levels of other select metabolites, including plate-activating factor (PAF) and PAF-like molecules, between Lassa fever and Ebola patients. Authenicate standards were used to confirm the identity of L-threonine and selected PAF-like molecules.

\section{Methods \\ Human subjects}

The Tulane University Institutional Review Board and the Sierra Leone Ethics and Scientific Research Committee original author(s) and the source, provide a link to the Creative Commons licence, and indicate if changes were made. The images or other third party material in this article are included in the article's Creative Commons licence, unless indicated otherwise in a credit line to the material. If material is not included in the article's Creative Commons licence and your intended use is not permitted by statutory regulation or exceeds the permitted use, you will need to obtain permission directly from the copyright holder. To view a copy of this licence, visit http://creativecommons.org/licenses/by/4.0/. The Creative Commons Public Domain Dedication waiver (http://creativeco mmons.org/publicdomain/zero/1.0/) applies to the data made available in this article, unless otherwise stated in a credit line to the data. 
approved this project. Patients were referred to the Kenema Government Hospital (KGH) Lassa Ward from the hospital's general ward or from regional health centers on the basis of suspicion of Lassa fever. Patients who met the case definition of Lassa fever as defined by Khan et al. [5] were admitted and cared for by the ward's trained staff. After the initial cases of Ebola were detected, patients were referred if they presented with an illness that met the World Health Organization case definition for Ebola. We obtained samples using the collection and processing protocols at Kenema Government Hospital under the emergency-response guidelines established by the Sierra Leone Ministry of Health and Sanitation. Diagnostic tests for the presence of Ebola virus (EBOV) were performed on site by means of quantitative reverse-transcriptasepolymerase chain reaction assays with the use of the SuperScript III One-Step RT-PCR System with Platinum Taq DNA Polymerase (Life Technologies).

\section{Serum processing for metabolomics analyses}

Small blood volumes (approximately $5 \mathrm{~mL}$ ) for serum separation were collected from patients presenting to KGH with febrile illnesses that met preclinical criteria of suspected Lassa fever or Ebola. Patient samples received a coded designation and were collected in serum vacutainer tubes. Blood samples were allowed to coagulate for $20 \mathrm{~min}$ at room temperature. Serum was separated from coagulated blood by centrifugation $(200 \times g, 20 \mathrm{~min}$ at room temperature). For subjects for which there was excess serum not needed for clinical evaluations, aliquots of the serum fraction were stored in cryovials at $-20{ }^{\circ} \mathrm{C}$ prior to processing for metabolite analysis.

Serum metabolite analysis was performed as previously described [1]. Briefly, serum samples were depleted of protein by addition to one part sera $(100 \mu \mathrm{L})$ of 4 parts ice-cold methanol $(400 \mu \mathrm{L})$, the mixture was vortexed vigorously for $10 \mathrm{~s}$, and incubated $1 \mathrm{~h}$ at $-20^{\circ} \mathrm{C}$ followed by centrifugation at $14,000 \times g, 15 \mathrm{~min}, 4^{\circ} \mathrm{C}$. The supernatant was collected and transferred to a new, sterile vial and dried under vacuum. The resultant smal L-molecule containing pellets were stored in desiccated, sealed containers and shipped to Tulane University where they were gamma-irradiated. Small molecule containing pellets were dissolved in a solution of 95:5 water:acetonitrile transferred to autosampler vials, and held at $-20^{\circ} \mathrm{C}$ or $4{ }^{\circ} \mathrm{C}$ immediately prior to analysis. All reagents utilized were high-pressure liquid chromatography (HPLC) grade.

\section{Liquid Chromatography Mass Spectrometry}

The Liquid Chromatography Mass Spectrometry (LCMS) method was performed as previously described with minor changes [1]. Briefly, detection of metabolites was performed via HPLC separation with ESI-MS (electrospray mass spectrometry) detection. HLPC was performed with an aqueous norma L-phase, hydrophilic interaction chromatography HPLC column: a Cogent Diamond Hydride Type-C column with $4 \mu \mathrm{m}$ particles and dimensions of $150 \mathrm{~mm}$ length and $2.1 \mathrm{~mm}$ diameter or an Agilent Zorbax 300-SB-C18 column with $3.0 \mu \mathrm{m}$ particles and dimensions of $150 \mathrm{~mm}$ length and $0.3 \mathrm{~mm}$ diameter was used with an Agilent 1290 HPLC system (Agilent Technologies, Santa Clara, CA). The column were maintained at $60{ }^{\circ} \mathrm{C}$ with a flow rate of $900 \mu \mathrm{L} /$ min. Chromatography was as follows: solvent consisted of $\mathrm{H}_{2} \mathrm{O}$ with $0.1 \%$ (v/v) formic acid for channel " $\mathrm{A}$ " and acetonitrile with $0.1 \%$ formic acid for channel "B". Following column equilibration at $98 \% \mathrm{~B}$, the sample was injected via autosampler, and the column was flushed for $2.0 \mathrm{~min}$ to waste. From $2.0 \mathrm{~min}$ to $14.5 \mathrm{~min}$, the gradient was linearly ramped from 98 to $65 \%$ B. From $14.5 \mathrm{~min}$ to $16.0 \mathrm{~min}$, the gradient was ramped from 65 to $25 \% \mathrm{~B}$. From 14.5 to $18.0 \mathrm{~min}$ the column was held at $25 \% \mathrm{~B}$, and from 18.0 to 18.2 min the gradient was ramped from 25 to $98 \%$ B. From 18.2 to $20.0 \mathrm{~min}$ the column was re-equilibrated with 98\% B. An Agilent 6538 Quad-Time of Flight with dua L-electron spray ion source mass spectrometer was used for all analyses. Resolution was approximately 20,000 and accuracy was $1 \mathrm{ppm}$. Source parameters: drying gas $12 \mathrm{~L} / \mathrm{min}$, nebulizer $60 \mathrm{psi}$, capillary voltage $3500 \mathrm{~V}$, capillary exit $100 \mathrm{~V}$. Spectra were collected in positive mode from 50 to $1700 \mathrm{~m} / \mathrm{z}$ at a rate of $1 \mathrm{~Hz}$.

\section{Molecular standards}

Authenticated standards of synthetic platelet-activating factor (PAF) C-16 (\#878110) and lysoPAF C-16 (\#878119) at a concentration of $5 \mathrm{mg} / \mathrm{mL}$ in chloroform were purchased from Avanti Polar Lipids (Birmingham, AL). L-threonine (T8625) was purchased from Sigma-Aldrich. The molecules were diluted in 95:5 water:acetonitrile solution and analyzed with the identical method for metabolite detection.

\section{Data analysis and visualization}

Raw spectral data in.d format where uploaded to XCMS Online (Versions 2.3.0 or 2.2.3) and processed as pairwise comparisons using parameters optimized for data acquired with UPLC on an Agilent 6538 MS.

\section{Statistics and machine learning}

Statistical analyses were carried out using the R statistical software package or Graphpad Prism. Multiple comparisons were performed by Analysis of Variance (ANOVA); $p<0.5$ was considered significant. Raw mass spectral intensity values and a unique identifier for specific 
spectral features were extrapolated from XCMS output and compiled into.csv file types.

\section{Results}

\section{Characteristics of a cohort of subjects presenting} to Kenema Government Hospital with Lassa fever or Ebola A panel of 50 serum samples from febrile patients triaged to a ward for the care of suspected Lassa fever patients was assembled to corroborate earlier observations of biomarkers of poor outcome in Lassa fever. Serum samples were drawn upon admittance and in all but two instances diagnostic tests were performed within $24 \mathrm{~h}$. Twentytwo subjects tested negative for Lassa virus (LASV) by all diagnostic tests and were classified as febrile non-Lassa. Subjects with a positive diagnosis for Lassa fever were subdivided into patients that were discharged $(\mathrm{n}=12)$ and those that succumbed to infection $(n=16)$. Gender and age data were available for 48 of the 50 samples. Subjects were $54 \%$ female $(26 / 48)$ with a mean age of 25.0 (years, range $<1-60$ ). There were 17 and 10 female and male LASV positive samples, respectively. Nine females and 6 males succumbed to Lassa fever with an average age of 21.5 (years, range $<1-38$ ) and an average time from symptom onset to death of 7.75 (days, 5-16). There were no significant differences between the distribution of LASV positive subjects or mortality between female and male subjects. A panel of serum samples derived from KGH collected during the 2015 EBOV outbreak in West Africa were also analyzed. Twelve febrile subjects tested negative for Lassa virus (LASV) and EBOV by all diagnostic tests and were classified as febrile nonEbola. Twenty-nine subjects tested positive for EBOV at the time of hospitalization and were classified as Ebola positive.

\section{L-Threonine is elevated in VHF patients}

Previously several unknown molecular features were identified in the blood of acutely ill Lassa fever patients [1]. Based on results from Eisfeld et al. [2] authentic standards were used to identify certain of the unknown molecules as adducts of $\mathrm{L}$-threonine (Fig. 1). Two features that were significantly elevated and detected as mass-to-charge ratio $(\mathrm{m} / \mathrm{z}) 102.0537$ and 119.0800 at identical retention times $[\mathrm{rt}=15.95]$. These features have now been identified as the $\mathrm{H}^{+} \mathrm{H}_{2} \mathrm{O}^{-}$and $\mathrm{NH}_{4}{ }^{+} \mathrm{H}_{2} \mathrm{O}^{-}$adducts of $\mathrm{L}$-threonine. L-threonine was significantly elevated in specimens from Ebola patients (Fig. 1a,b). In contrast, L-threonine was not elevated in the blood of convalescent Ebola patients or blood samples from non-febrile controls. Likewise, L-threonine was significantly elevated in specimens

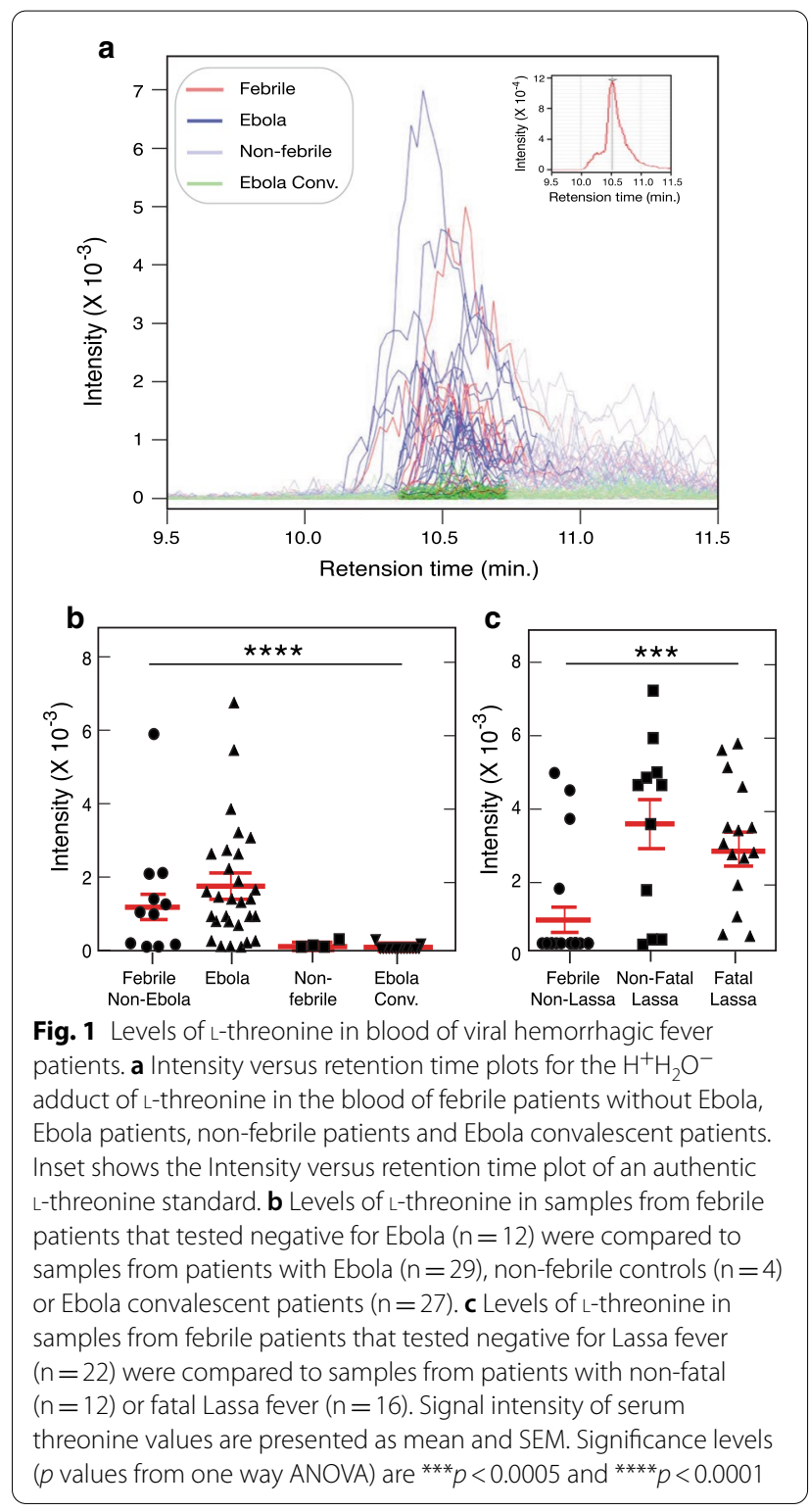

from Lassa fever patients compared to febrile subjects without Lassa fever (Fig. 1c). No significant different was observed between Lassa fever subjects that succumbed to Lassa fever and Lassa fever survivors. Most febrile patients that presented to the VHF Ward at $\mathrm{KGH}$ and tested negative for Ebola or Lassa fever had low blood levels of L-threonine. However, a subset of febrile patients in both the Ebola (Fig. 1b) and Lassa cohorts (Fig. 1c) had elevated blood L-threonine. These results show that elevated L-threonine may be a common marker of acute VHFs as patients positive for both EBOV and LASV infection have significant elevation of this compound in the blood across spatially and temporally distinct disease outbreaks. 
Platelet-activating factor and platelet-activating factor like-lipids are decreased in subjects with fatal Lassa fever Platelet activity is depressed during Lassa fever, particularly in terminal patients $[6,7]$. Twenty-four PAF or PAFlike molecules were putatively identified and expressed at variable levels in the serum of febrile patients presenting to KGH (Fig. 2). The cluster analysis indicated that nearly all PAFs or PAF-like molecules were present in lower amounts in the serum of patients with fatal Lassa fever than in patients that survived the acute infection (nonfatal Lassa fever). Non-Lassa febrile illness patients had the highest overall levels of PAF or PAF-like molecules. We also positively confirmed the identity of PAF and lysoPAF with LCMS versus authenticated standards in the serum of patients with Lassa fever. PAF and PAF-like molecules are reduced Lassa fever patients that have a fatal outcome. These lipids were not observed to be similarly dysregulated in a limited cohort of serum samples of Ebola positive patients compared to convalescent Ebola and febrile non-Ebola patients (Additional file 1: Fig. S1).

\section{Discussion}

A previously unknown molecule elevated in fatal, acute, and post-Lassa acute febrile patients has been identified as L-threonine. The serum of Ebola patients also has elevated levels of L-threonine. Results showing elevation of L-threonine in the blood of Lassa fever and Ebola patients confirms and extend results previously published by Eisfeld and coworkers [2]. These investigators demonstrated that levels of serum L-threonine, as well as a vitamin D binding protein, perfectly stratified Ebola patients by outcome, providing better predictive ability than viral load. Their results prompted us to use authentic standards to identity L-threonine as a Lassa fever biomarker. The use of authenic standards for selected PAF-like molecules also corroborates and improves the results of our prior metabolomic analyses [1].

Prior metabolomic studies have identified L-threonine as a marker of infection by several viral and nonviral pathogens [2-4]. The amniotic fluid of human cytomegalovirus infected (HCMV) women shows elevated levels of L-threonine compared to non-HCMV infected women [8]. No significance difference in vertical transmission of HCMV based on elevated L-threonine was observed. Glycine, serine, and threonine metabolic pathways were found to be altered in chikungunya or dengue patients [9]. In contrast to prior results in Ebola patients [2], L-threonine was elevated in both Lassa fever patients that succumbed to their illness as well as subjects that survived. Further studies are required to determine whether elevated serum L-threonine represents a general marker of VHF or other severe viral infections. We confirmed that PAF and molecules with PAF moiety are reduced in the blood of patients with fatal Lassa fever. However, similar changes were not observed in the blood of Ebola patients. Additional studies on the different roles for PAF and PAF-like molecules in severe viral diseases should be conducted.

\section{Conclusions}

Metabolomics may provide tools to identify pathways that are differentially affected during VHFs. For patients suffering from VHF it would also be advantageous to have a measure of disease progression/severity, such as L-threonine levels, to predict outcomes several days prior to death. Coupling the detection and changes of these and other analytes with appropriate rapid diagnostics through disease progression may serve as a mechanism to

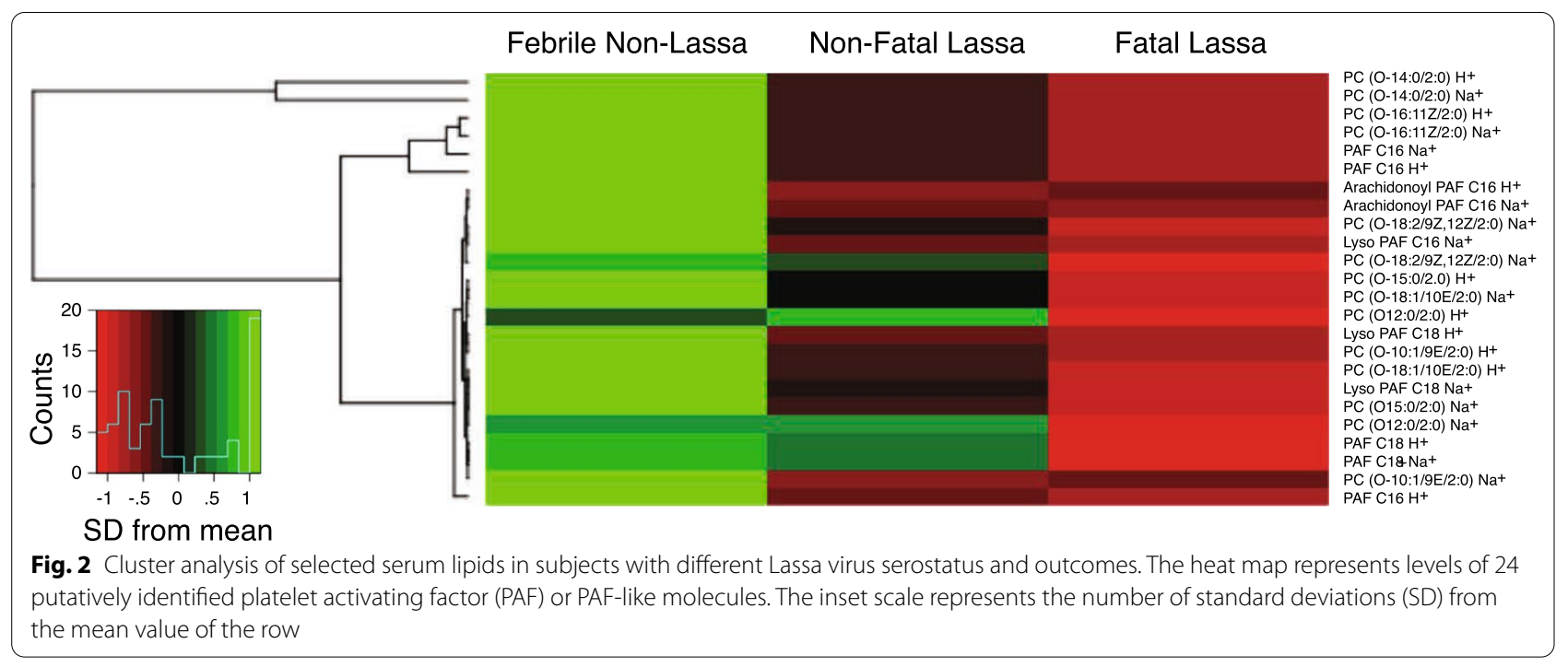


monitor and predict outcome, ensuring scarce resources are allocated where needed most.

\section{Supplementary information}

Supplementary information accompanies this paper at https://doi. org/10.1186/s12985-020-01459-y.

Additional file 1: Figure S1. Levels of selected platelet-activating factor and platelet-activating factor-like molecules in the blood of Lassa fever and Ebola patients.

\section{Abbreviations}

VHF: Viral hemorrhagic fever; KGH: Kenema Government Hospital; EBOV: Ebola virus; LASV: Lassa virus; m/z: Mass-to-charge ratio; HPLC: High-pressure liquid chromatography; LCMS: Liquid Chromatography Mass Spectrometry; HCMV: Human cytomegalovirus infected; PAF: Platelet-activating factor; ANOVA: Analysis of variance; VHFC: Viral Hemorrhagic Fever Consortium.

\section{Acknowledgments}

We thank the other members of the Viral Hemorrhagic Fever Consortium (VHFC): The Broad Institute, Harvard University, La Jolla Institute of Immunology, the Scripps Research Institute, Tulane University, the University of Texas Medical Branch, Zalgen Labs, the ISTH Lassa Fever Program, Redeemer's University and $\mathrm{KGH}$ ) without which this work could not have been conducted; and the patients with serious febrile illnesses who presented to $\mathrm{KGH}$, as well as their families, without whose cooperation this study would not have been possible. Simbirie C. Jalloh, Allyson M. Haislip, Christopher M. Bishop, Tynette D. Hills, and Douglass T. Simpson provided program management and logistical support.

\section{Authors' contributions}

Conceptualization, TVG; Methodology, TVG, JSS, LMB, DSG; Investigation, TVG, JSS, LMB, RFG, DSG; Writing—Original Draft, TVG; Writing-TVG LMB, RFG. DSG; Funding Acquisition, JSS, LMB, RFG. DSG; Resources, JSS, LMB, RFG. DSG; Supervision, RFG.

\section{Funding}

Funding support came from NIH Grants Al104621, Al114855, Al1 15754, and 1U19Al135995. The funders had no role in the collection, analysis, and interpretation of data or in writing the manuscript.

\section{Availability of data and materials}

Data has been deposited in the XCMS Public archive (https://xcmsonline .scripps.edu/landing_page.php?pgcontent=mainPage) under the identifier: 1181466.

\section{Ethics approval and consent to participate}

The Tulane University Institutional Review Board (191330) and the Sierra Leone Ethics and Scientific Research Committee (070716) approved this project.

\section{Consent to publication}

Not applicable.

\section{Competing interests}

JSS, LMB, RFG and DSG are members of the VHFC (www.vhfc.org). The VHFC is a partnership of academic and industry scientists who are developing diagnostic tests, therapeutic agents, and vaccines for Lassa fever, Ebola, and other severe diseases. Tulane University and its various academic and industry partners have filed US and foreign patent applications on behalf of the consortium for several of these technologies. Technical information may also be kept as trade secrets. If commercial products are developed, consortium members may receive royalties or profits. This does not alter our adherence to all policies of the NIH and Virology Journal on sharing data and materials. Financial and non-financial competing interests that the editors consider relevant to the content of the manuscript have been disclosed. RFG and LMB are co-founders of Zalgen. LMB is a Zalgen employee. All other authors declare no competing interests.

\section{Author details}

${ }^{1}$ Department of Microbiology and Immunology, Tulane University, 1430 Tulane Avenue, JBJ568, New Orleans, LA 70112, USA. ${ }^{2}$ Sections of Infectious Disease, Departments of Pediatrics and Internal Medicine, School of Medicine, Tulane University, New Orleans, LA, USA. ${ }^{3}$ Zalgen Labs, LLC, Germantown, MD, USA. ${ }^{4}$ Viral Hemorrhagic Fever Program, Kenema Government Hospital, Kenema, Sierra Leone. ${ }^{5}$ Ministry of Health and Sanitation, Freetown, Sierra Leone.

${ }^{6}$ Present Address: Ansun Biopharma, San Diego, CA 92121, USA.

Received: 2 October 2020 Accepted: 23 November 2020

Published online: 26 November 2020

References

1. Gale TV, Horton TM, Grant DS, Garry RF. Metabolomics analyses identify platelet activating factors and heme breakdown products as Lassa fever biomarkers. PLoS Negl Trop Dis. 2017;11(9):e0005943.

2. Eisfeld AJ, Halfmann PJ, Wendler JP, Kyle JE, Burnum-Johnson KE, Peralta Z, Maemura T, Walters KB, Watanabe T, Fukuyama S, et al. Multi-platform 'Omics analysis of human Ebola virus disease pathogenesis. Cell Host Microbe. 2017;22(6):817-829.e818.

3. Weiner J 3rd, Parida SK, Maertzdorf J, Black GF, Repsilber D, Telaar A, Mohney RP, Arndt-Sullivan C, Ganoza CA, Faé KC, et al. Biomarkers of inflammation, immunosuppression and stress with active disease are revealed by metabolomic profiling of tuberculosis patients. PLOS ONE. 2012;7(7):e40221.

4. Abdelrazig S, Ortori CA, Davey G, Deressa W, Mulleta D, Barrett DA, Amberbir A, Fogarty AW. A metabolomic analytical approach permits identification of urinary biomarkers for Plasmodium falciparum infection: a case-control study. Malar J. 2017;16(1):229.

5. Khan SH, Goba A, Chu M, Roth C, Healing T, Marx A, Fair J, Guttieri MC, Ferro $P$, Imes T, et al. New opportunities for field research on the pathogenesis and treatment of Lassa fever. Antivir Res. 2008;78(1):103-15.

6. Lange JV, Mitchell SW, McCormick JB, Walker DH, Evatt BL, Ramsey RR. Kinetic study of platelets and fibrinogen in Lassa virus-infected monkeys and early pathologic events in Mopeia virus-infected monkeys. Am J Trop Med Hyg. 1985;34(5):999-1007.

7. Cummins D, Fisher-Hoch SP, Walshe KJ, Mackie IJ, McCormick JB, Bennett D, Perez G, Farrar B, Machin SJ. A plasma inhibitor of platelet aggregation in patients with Lassa fever. Br J Haematol. 1989;72(4):543-8.

8. Fattuoni C, Palmas F, Noto A, Barberini L, Mussap M, Grapov D, Dessì A, Casu M, Casanova A, Furione M, et al. Primary HCMV infection in pregnancy from classic data towards metabolomics: an exploratory analysis. Clin Chim Acta Int J Clin Chem. 2016;460:23-32.

9. Shrinet J, Shastri JS, Gaind R, Bhavesh NS, Sunil S. Serum metabolomics analysis of patients with chikungunya and dengue mono/co-infections reveals distinct metabolite signatures in the three disease conditions. Sci Rep. 2016;6:36833.

\section{Publisher's Note}

Springer Nature remains neutral with regard to jurisdictional claims in published maps and institutional affiliations. 